Article

\title{
Poly(Phenylene Methylene): A Multifunctional Material for Thermally Stable, Hydrophobic, Fluorescent, Corrosion-Protective Coatings
}

\author{
Marco F. D'Elia ${ }^{1}$, Andreas Braendle 1, Thomas B. Schweizer ${ }^{1}$, Marco A. Ortenzi 2 (D), \\ Stefano P. M. Trasatti ${ }^{3}$, Markus Niederberger ${ }^{1}$ and Walter Caseri ${ }^{1, *}$ (ID) \\ 1 Department of Materials, ETH Zurich, 8093 Zürich, Switzerland; marco.delia@mat.ethz.ch (M.F.D.); \\ andreas.braendle@mat.ethz.ch (A.B.); thomas.schweizer@mat.ethz.ch (T.B.S.); \\ markus.niederberger@mat.ethz.ch (M.N.) \\ 2 Department of Chemistry, University of Milan, 20133 Milan, Italy; marco.ortenzi@unimi.it \\ 3 Department of Environmental Science and Policy, University of Milan, 20133 Milan, Italy; \\ stefano.trasatti@unimi.it \\ * Correspondence: walter.caseri@mat.ethz.ch
}

Received: 29 June 2018; Accepted: 25 July 2018; Published: 7 August 2018

\begin{abstract}
Poly(phenylene methylene) (PPM) is a thermally stable, hydrophobic, fluorescent hydrocarbon polymer. PPM has been proposed earlier to be useful as a coating material but this polymer was isolated in relevant molar masses only recently, and in large quantities. Accordingly, the preparation of coatings based on PPM and their behavior was explored in this study, with the example of the metal alloy AA2024 as a common substrate for corrosion tests. Coatings free of bubbles and cracks were obtained by hot pressing and application of the following steps: Coating on AA2024 with a layer of polybenzylsiloxane to improve the adhesion between PPM and the metal surface, the addition of polybenzylsiloxane to PPM in order to enhance the viscosity of the molten PPM, and the addition of benzyl butyl phthalate as a plasticizer. Electrochemical corrosion tests showed good protection of the metal surface towards a $\mathrm{NaCl}$ solution, thanks to a passive-like behavior in a wide potential window and a very low current density. Remarkably, the PPM coating also exhibited self-healing towards localized attacks, which inhibits the propagation of corrosion.
\end{abstract}

Keywords: poly(phenylene methylene), aluminum alloy; AA2024; coatings by hot pressing; additives; fluorescence; corrosion protection

\section{Introduction}

Poly(phenylene methylene) (PPM) (Figure 1a) is a hydrocarbon polymer which consists of an alternating sequence of phenylene and methylene units. Therefore, PPM is structurally located between polyethylene and polyphenylene. Usually, the PPM products synthesized so far contain a mixture of ortho-, meta- and para-substituted phenylene units, with the latter reported to be dominant [1-3]. Interestingly, PPM unexpectedly exhibits photoluminescence, although the phenylene rings are not conjugated as in common fluorescent organic polymers [4]. This property was ascribed to homoconjugation, a rare phenomenon which can arise under certain geometrical conditions when conjugated $\pi$-orbital systems interact with each other, although they are separated by an electronically insulating group such as a methylene group [5,6], as illustrated for PPM in Figure 1b. 


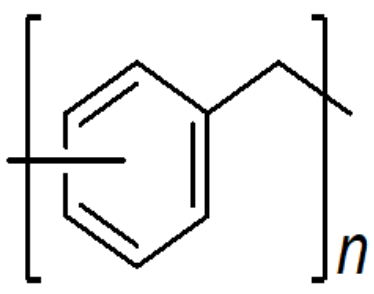

(a)

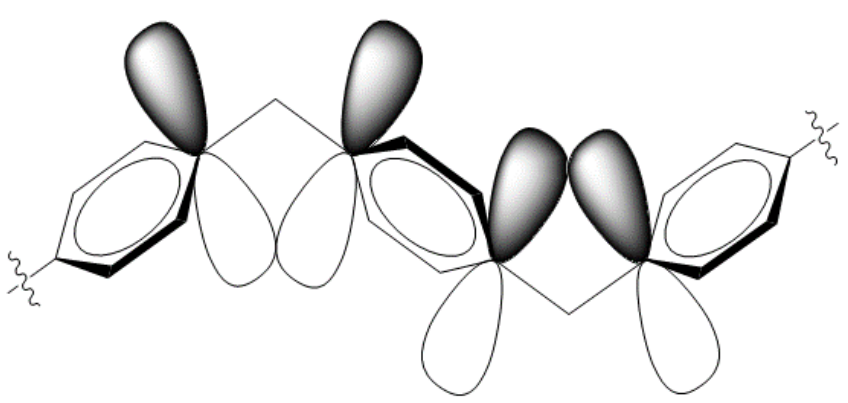

(b)

Figure 1. (a) Chemical structure of poly(phenylene methylene) (PPM); (b) Schematic representation of homoconjugation in PPM as a result of overlapping p-orbitals of phenylene rings which are separated by a methylene group.

Remarkably, in addition to hydrophobicity, PPM shows exceptionally high thermal stability [7-13] (onset of decomposition at $470{ }^{\circ} \mathrm{C}$ by thermogravimetric analysis under air atmosphere [4]). Further, PPM is structurally similar to phenol resins but possesses higher thermal stability [14]. Accordingly, PPM has been proposed as a valuable material for coatings [15-18]. In the panorama of protective coatings against corrosion of polymers with similar application methods and protection mechanism of PPM, there are epoxy resins, especially FBE formulations, and epoxy-polyaniline systems. These two classes of compounds present thermal stability of about $300^{\circ} \mathrm{C}$ for epoxides, and about $200-240{ }^{\circ} \mathrm{C}$ for polyaniline $[19,20]$. Moreover, coatings with these polymers are not fluorescent and are also expected to be less hydrophobic than PPM, due to their chemical structures. In light of this, in addition to the high thermal stability of PPM $\left(450-470^{\circ} \mathrm{C}\right)$, PPM presents a unique combination of properties, such as fluorescence and hydrophobicity, where the latter can markedly influence the features of corrosion protective coatings.

However, coatings with PPM have not been investigated yet, presumably as PPM with adequate molar masses (number average molar mass $M_{\mathrm{n}} 10,000 \mathrm{~g} \cdot \mathrm{mol}^{-1}$ ) has been isolated only recently $[4,21]$. Obviously, in the previously described synthesis procedures crucial conditions such as application of a steady nitrogen flow to remove the evolving hydrochloric acid and bulk polymerization under mechanical stirring were not adequately incorporated. Such parameters are even more important when polymerization proceeds via a step growth mechanism, as in the case of catalysts like $\mathrm{SnCl}_{4}$. Notably, the synthesis of PPM is straightforward and it can readily be produced in large quantities (>100 g) on the laboratory scale.

Hence, in this work the preparation of coatings with PPM is explored. This includes the identification of a suited coating method and associated conditions for the maunfacture of coatings. Since it turned out in the course of the experiments that the film quality could be improved by additives, this aspect is also addressed. An aluminum alloy, AA2024, was selected as the substrate, pre-treated to enhance the coating adhesion, and used for corrosion tests. Accordingly, the ability of PPM in anti-corrosion protection is also examined in this study, revealing unusual self-healing properties.

\section{Materials and Methods}

Benzyl chloride (99\%), tin(IV) chloride, phosphoric acid (85\%), chloroform, and octaphenyl silsesquioxane (OP-POSS) was purchased from Sigma Aldrich (Buchs, Switzerland), benzyltriethoxysilane from Fluorochem (Hadfield, UK), and methanol (98\%) from Merck (Darmstadt, Germany).

Poly(phenylene methylene) was synthesized by polymerization of benzyl chloride with $\mathrm{SnCl}_{4}$ as a catalyst according to the literature [21], with number average molar mass $M_{\mathrm{n}}=11,100 \mathrm{~g} \cdot \mathrm{mol}^{-1}$ and weight average molar mass $M_{\mathrm{W}}=34,200 \mathrm{~g} \cdot \mathrm{mol}^{-1}$, determined by gel permeation chromatography (GPC).

The common aluminum alloy AA2024 (also designated as Al-2024, containing 4.3\%-4.5\% copper, $0.5 \%-0.6 \%$ manganese, $1.3 \%-1.5 \%$ magnesium and less than $0.5 \%$ of other elements) was used as a 
substrate. Sheets of $12 \mathrm{~cm}$ in length, $3 \mathrm{~cm}$ in width and $4 \mathrm{~mm}$ in thickness were provided by Aviometal s.p.a (Varese, Italy). Samples of $4 \mathrm{~cm}$ in length were cut and subsequently polished with abrasive papers of 300, 500, 800 and 1200 grit. Immediately after polishing, the samples were cleaned by immersion in ethanol in an ultrasonic bath (Banderlin, Berlin, Germany) for $5 \mathrm{~min}$. Then the samples were removed from the ethanol bath and the residual ethanol at the surface of the AA2024 samples was evaporated by means of a flush of nitrogen.

The freshly cleaned samples were covered with a layer of benzyltriethoxysilane by spin coating. Initially, $0.3 \mathrm{~mL}$ neat benzyltriethoxysilane was deposited in the center of the AA2024 substrates with a micropipette. Thereafter the spinning rate was increased from 0 to $3500 \mathrm{rpm}$. and maintained at this rate for $30 \mathrm{~s}$. Finally, the samples were heated to $100{ }^{\circ} \mathrm{C}$ for $1 \mathrm{~min}$, whereupon condensation of benzyltriethoxysilane to respective polysiloxanes commonly proceeds as described below. These samples were coated with PPM in absence and presence of siloxanes (OP-POSS and PBS, acronym of PBS see below) and plasticizer by hot pressing, as described in the section Results and Discussion, using about $100 \mathrm{mg}$ of coating material.

A soluble condensation product of benzyltriethoxysilane, i.e., a polybenzylsiloxane designated as PBS, was synthesized under nitrogen atmosphere. A quantity of $0.40 \mathrm{~mL}$ ( $5.9 \mathrm{mmol}$ ) of phosphoric acid, $0.2 \mathrm{~mL}(10 \mathrm{mmol})$ of deionized water and $2.98 \mathrm{~g}(12 \mathrm{mmol})$ of benzyltriethoxysilane were transferred into a $100 \mathrm{~mL}$ three-neck flask equipped with a mechanical glass stirrer. The reaction was performed at room temperature, and after $10 \mathrm{~min}$, a sol-gel reaction led to the formation of a highly viscous white material. After $24 \mathrm{~h}$, during which evaporation of the reaction byproduct ethanol most likely took place under nitrogen flush, the material became an odourless white solid $(2.43 \mathrm{~g})$. A part, $1 \mathrm{~g}$ of the material, was dissolved in $5 \mathrm{~mL}$ of acetone, precipitated into $200 \mathrm{~mL}$ of water, filtered (cellulose filter) and dried under vacuum $\left(10^{-2}\right.$ mbar, $\left.12 \mathrm{~h}\right)$ to obtain $0.78 \mathrm{~g}$ of PBS. ${ }^{1} \mathrm{H}-\mathrm{NMR}$ spectra did not show anymore the characteristic $-\mathrm{O}-\mathrm{CH}_{2}-\mathrm{CH}_{3}$ signal of ethoxy groups (triplet at $1.24 \mathrm{ppm}$, quadruplet at $3.83 \mathrm{ppm}$ ), i.e., the ethoxy groups initially present in benzyltriethoxysilane were virtually completely converted to ethanol under formation of siloxane units. Elemental analysis of the product (in $\% \mathrm{~m} / \mathrm{m}$ ): C 55.86, H 5.01.

Polymerization of benzyl chloride in presence of siloxanes was performed as follows: Under nitrogen atmosphere, $12 \mathrm{~mL}$ (104 mmol) of destabilized benzyl chloride was given to $0.132 \mathrm{~g}$ of additive (OP-POSS or PBS) in a $100 \mathrm{~mL}$ three-neck flask equipped with mechanical stirrer. After heating the mixture to $80^{\circ} \mathrm{C}, 0.04 \mathrm{~mL}(0.34 \mathrm{mmol})$ of $\mathrm{SnCl}_{4}$ was added. Polymerization was carried out under a constant nitrogen flow of $0.4-0.5 \mathrm{~mL} \cdot \mathrm{min}^{-1}$ to allow the produced $\mathrm{HCl}$ to escape from the reaction batch. After $3 \mathrm{~h}$, the temperature was risen to $120^{\circ} \mathrm{C}$ for $1 \mathrm{~h}$ and subsequently, due to the increase of viscosity upon polymerization, to $180^{\circ} \mathrm{C}$ for $20 \mathrm{~h}$. During the reaction, the color changed from deep red at the beginning of the reaction to a clear amber at the end of the reaction. The resulting reaction mixture was allowed to cool down to room temperature. An aliquot of $1 \mathrm{~g}$ of product was removed from the solids and dissolved in $5 \mathrm{~mL}$ of chloroform. This solution was poured into $250 \mathrm{~mL}$ methanol under stirring, filtered (cellulose filter) and dried under vacuum $\left(10^{-2}\right.$ mbar) for $12 \mathrm{~h}$. The following quantities of product were obtained (the origin of the acronyms becomes evident in the section Results): $0.83 \mathrm{~g}$ of H-OP-POSS(P) and $0.74 \mathrm{~g}$ of H-PBS(P). The number average molar mass $\left(M_{n}\right)$ of both polymers was in the range of 9000 to $11,000 \mathrm{~g} \mathrm{~mol}^{-1}$.

For experiments with PPM comprising additives mixed in solution (PBS) or dispersion (OP-POSS), respectively, $3.00 \mathrm{~g}$ of PPM (synthesis according to the literature, see above) were stirred in $20 \mathrm{~mL}$ chloroform together with $0.03 \mathrm{~g}$ of siloxane (OP-POSS or PBS). The mixture was poured into $250 \mathrm{~mL}$ of methanol, and the precipitated material was filtered and dried at $10^{-2}$ mbar for $12 \mathrm{~h}$. Yields for the products designated as H-OP-POSS(D): $2.96 \mathrm{~g}$, H-PBS(D): $2.74 \mathrm{~g}$.

Materials with plasticizer were produced starting with $200 \mathrm{mg}(0.64 \mathrm{mmol})$ of benzyl butyl phthalate (BBP) which was added to $1.2 \mathrm{~g}$ of H-PBS(D) in $2 \mathrm{~mL}$ of tetrahydrofuran (THF) under magnetic stirring. When PPM and plasticizer were molten, the THF was evaporated at $60{ }^{\circ} \mathrm{C}$ under 
vacuum for $1 \mathrm{~h}$ and subsequently at room temperature under vacuum for $8 \mathrm{~h}$. A quantity of $1.378 \mathrm{~g}$ product was obtained.

For rheology measurements, an Anton-Paar MCR-302 rheometer with parallel plates (Graz, Austria) was used, at a temperature of $90^{\circ} \mathrm{C}$. The values of complex viscosity, storage modulus and loss modulus were measured by collecting 20 points at angular frequencies starting from 400 to $0.4 \mathrm{~s}^{-1}$.

Electrochemical characterizations were performed on AA2024 samples surface-modified with condensed benzyltriethoxysilane (see above) and a layer of H-PBS(D). The experiments were conducted in a simulated marine environment, i.e., at $\mathrm{pH} 6.9 \pm 0.2$ and $0.6 \mathrm{~mol} \cdot \mathrm{L}^{-1} \mathrm{NaCl}$ (p.a., $99.8 \%$, Sigma-Aldrich, Buchs, Switzerland) prepared with MilliQ water. The temperature was equal for all experiments $\left(21.1 \pm 1.0^{\circ} \mathrm{C}\right)$.

A classic saturated calomel electrode ( $\mathrm{SCE}=+242 \mathrm{mV}$ vs. SHE) was used for all experiments. Potentiodynamic polarization curves were recorded after an initial delay time of $600 \mathrm{~s}$ for equilibration at open circuit potential (OCP), on a surface area of $1 \mathrm{~cm}^{2}$ and at a rate of $0.167 \mathrm{mV} \cdot \mathrm{s}^{-1}$.

\section{Results}

\subsection{Surface-Modified Metal Substrates}

The high strength aluminum alloy AA2024 (also designated as Al-2024), widely employed in aerospace applications, was used as a substrate in this study. Due to its heterogeneous microstructure, this alloy is very sensitive to localised corrosion, particularly in chloride-containing environments [22]. These aluminum alloys were cleaned as described in the section Materials and Methods. When PPM films were prepared directly on cleaned AA2024, cracks arose in the final films within seconds after preparation, and the PPM readily detached from the surface (the same phenomenon occurred also when siloxanes were present in PPM as addressed below). In order to improve compatibility between the hydrophilic surface of the aluminum alloy and the hydrophobic PPM, the metal substrates were first covered by a layer of condensed benzyltriethoxysilanes of about $1 \mu \mathrm{m}$ thickness (determined by SEM from cross sections). This strategy has been established already long ago for corresponding purposes $[23,24]$. As the $\mathrm{Si}-\mathrm{OEt}(\mathrm{Et}=$ ethyl) bonds in the ethoxysilanes are sensitive to water (including ambient humidity) it is generally assumed that highly crosslinked polysiloxanes (here polybenzylsiloxanes) are formed as hydrolysis products at the surfaces of the substrates under release of ethanol. Indeed, the polybenzylsiloxane layer prevented detachment of the PPM-based coatings applied as described below.

\subsection{Coatings}

Coatings of PPM were produced by pressing powders of PPM onto silane-pretreated metal substrates, with a polyetheretherketone (PEEK) foil to separate the PPM from the pressing instrument. Pressing was performed at $90{ }^{\circ} \mathrm{C}$, i.e., above the glass transition temperature of the polymer (around $65^{\circ} \mathrm{C}$ ), for $30 \mathrm{~s}$ (no significant difference in the quality of the coatings was found by pressing for $60 \mathrm{~s}$ ). The applied pressure amounted to $4 \mathrm{kPa}$. At lower pressures, the films were less uniform, and at higher pressures (up to $10 \mathrm{kPa}$ ) there was a tendency of enhanced formation of cracks in the final coatings. The thickness of the resulting films was about $40 \mu \mathrm{m}$ (determined by SEM from cross sections and surface profilometry), and the coatings appeared very unniform and homogeneous

In the PPM films prepared by hot pressing, some cracks always arose upon cooling to room temperature even under optimum pressing conditions (Figure 2), and a few bubbles were usually found in the films, too. Deposition of PPM from solution (e.g., from chloroform or tetrahydrofuran) followed by solvent evaporation was also investigated but the films thus obtained contained much more bubbles, which could not be eliminated. It is known that an increase in the viscosity of molten polymers (as is the case with PPM in the hot press) can reduce bubble formation and prevent cracks [25-27]. The viscosity of PPM might be enhanced by well dispersed aromatic polynuclear siloxanes which are compatible 
with PPM. The aromatic units of these siloxanes might interact with the aromatic moieties of PPM and thus hamper the flow of the polymer chains. Therefore, the application of corresponding siloxanes was explored for the reduction of cracks and bubbles. These siloxanes were octaphenyl polysilsesquioxane (OP-POSS) and a polycondensation product of benzyltriethoxyxsilane (PBS). The OP-POSS molecules possess a core of rather cubic shape with 8 silicon atoms at the adges of the cube. Neighoring silicon atoms are connected by an oxygen atom, and a phenyl group is attached to each silicon atom. The employed OP-POSS was a commercial product, the other comopound was synthesized as described in the section Materials and Methods. The synthesis method was optimized to obtain virtually complete conversion of the ethoxy groups of the starting material and complete solubility of the condensation product in chloroform. Preliminary experiments revealed that a concentration of $1 \%$ $\mathrm{m} / \mathrm{m}$ of siloxane was suited to improve the quality of PPM-based coatings.

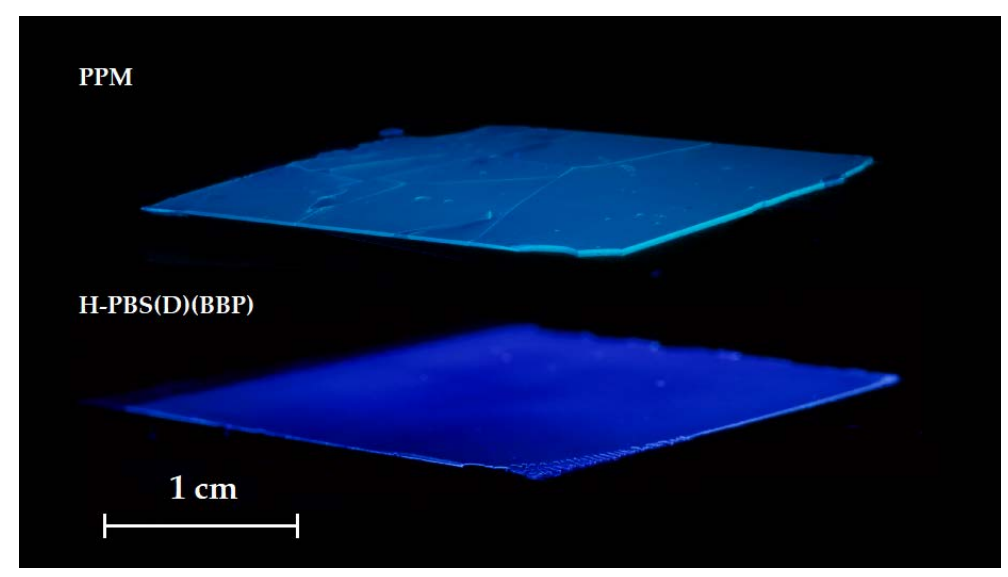

Figure 2. Top panel: PPM coating on polybenzylsiloxane-modified AA2024, showing cracks after the hot-pressed coating cooled down to room temperature. Bottom panel: PPM coating containing PBS and BBP (H-PBS(D)(BBP)), 2 weeks after hot-pressing. The pictures were taken irradiating with light having a wavelength of $365 \mathrm{~nm}$, causing the characteristic blue fluorescence of PPM.

In order to incorporate the siloxanes, two methods were employed. On the one hand, the monomer benzyl chloride was mixed with the siloxanes $(1 \% \mathrm{~m} / \mathrm{m})$ and polymerization was subsequently performed under the same conditions as for the synthesis of PPM itself. The siloxanes did not influence the course of polymerization significantly, in particular similar molar masses of PPM were obtained as in absence of siloxanes $\left(M_{\mathrm{n}}\right.$ about $10,000 \mathrm{~g} \mathrm{~mol}^{-1}$, see Materials and Methods). Also, the PPM with the siloxanes showed the common fluorescence of PPM (cf. Figure 2), and the glass transition temperature $\left(57^{\circ} \mathrm{C}\right.$, measured by DSC) was not significantly affected in all cases. The resulting material was also used directly for subsequent investigations without further work up. i.e., the mass ratio of additive and PPM necessarily remained in the order of the initial ratio of $1 \% \mathrm{~m} / \mathrm{m}$.

On the other hand, additives were introduced in PPM by mixing PPM solutions in chloroform with optically clear solutions of PBS and PBS-DS or opaque dispersions of OP-POSS. After precipitation in methanol, the resulting solids were filtered off. Although PPM as well as the siloxanes is insoluble in methanol, it cannot be excluded that the ratio of siloxane and PPM deviated from the initial ratio of $1 \% \mathrm{~m} / \mathrm{m}$. As a side note, preparation of films by evaporation of PPM solutions was generally not successful because upon solvent evaporation in the open atmosphere, a solid skin formed on top of the liquid (within about an hour in the case of chloroform). As a consequence, the solution underneath was trapped and the samples hardly dried (in the case of chloroform, they were not dry yet after a week), indicating very good barrier properties of PPM. Acceleration of evaporation by application of a vacuum lead to immense foaming.

Since the viscosity is considered to be an important factor for the preparation of crack-free films (see above), the complex viscosity and the damping factor (ratio of loss modulus and storage 
modulus) were determined. The hybrid-like materials containing PPM and PBS or OP-POSS are designated as H-PBS(D) or H-OP-POSS(D) when prepared from dispersion or solution and H-PBS(P) or H-OP-POSS(P) when prepared via in-situ polymerization of benzyl chloride. The complex viscosities of these materials are plotted against angular frequency in Figure 3. For all materials containing siloxanes higher values of complex viscosity were obtained compared to pure PPM (the molar masses of PPM were similar in all cases, $M_{\mathrm{n}}$ ca. $10,000 \mathrm{~g} \cdot \mathrm{mol}^{-1}$ ). A particularly large increase of the complex viscosity was observed for the material H-PBS(D), compared to the polymer H-PBS(P) (increase by a factor 10-15, depending on the angular frequency) and PPM (increase by a factor 10-25). A retention of the shear thinning behavior is evident by the high increase of viscosity at higher angular frequencies (between $50-100 \mathrm{rad} \cdot \mathrm{s}^{-1}$ ), and plateau values were often evident at lower frequencies. In the case of materials obtained using OP-POSS the complex viscosity of the material prepared by the dispersion approach was below that prepared by the polymerization approach (as a remark, OP-POSS did not dissolve well in the solvent used to dissolve PPM for the preparation of H-OP-POSS(D)). The viscosity of H-OP-POSS(D), however, did not increase to the extent of that of H-PBS(D).

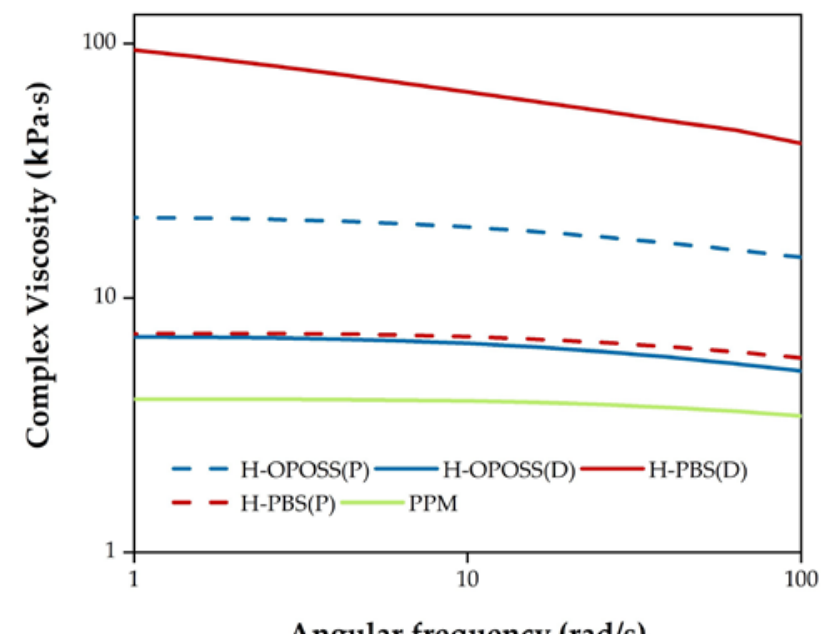

Figure 3. Complex viscosity of PPM without (green curve) and with additives (curves with other colors), as indicated in the figure.

Viscoelastic materials exhibit both elastic behavior and viscous behavior, respectively known in rheology as storage modulus and loss modulus. In order to determine how these parameters are balanced in PPM with siloxanes, values of damping factors were collected upon variation of the angular frequency in the same rheological experiment described above. In general, the addition of siloxane caused a decrease of the damping factor compared to pure PPM (Figure 4). With the exception of H-OP-POSS(D), however, the damping factors were above 1, implying the retention of the liquid-like structure in these cases. This indicates an absence of entanglements between siloxanes and PPM (as well as an absence of crosslinking but crosslinking is hardly possible with regard to the chemical nature of PPM and the siloxanes). According to the absence of chemical bonds between PPM and siloxanes these materials can be attributed to the so-called class 1 hybrid materials [28] that present high viscosity preserving a liquid-like structure in the molten phase, providing a suitable material for coating technologies. Yet for H-OP-POSS(D) the damping factor was far below 1, even though entanglements are highly improbable regarding the spheric structure of OP-POSS. Maybe segregation of OP-POSS in the polymer matrix can influence the rheologic measurement leading to these unexpected data. Notably, OP-POSS did not dissolve in chloroform but was present as an opaque dispersion upon mixing with the PPM solution, which implies the presence of OP-POSS aggregates. On the other hand, formation of a percolated network cannot be excluded. 
The rather abrupt increase of the damping factor at frequencies below ca. $1 \mathrm{rad} \cdot \mathrm{s}^{-1}$ (Figure 4 ) could essentially originate in a sol-gel-like transition. At high frequency the polymer forms a gel-like state or percolation network, while at low frequency the polymer is in a quasi-liquid state, i.e., the polymer molecules have sufficient time to move freely with respect to each other and can thus balance the external stress.

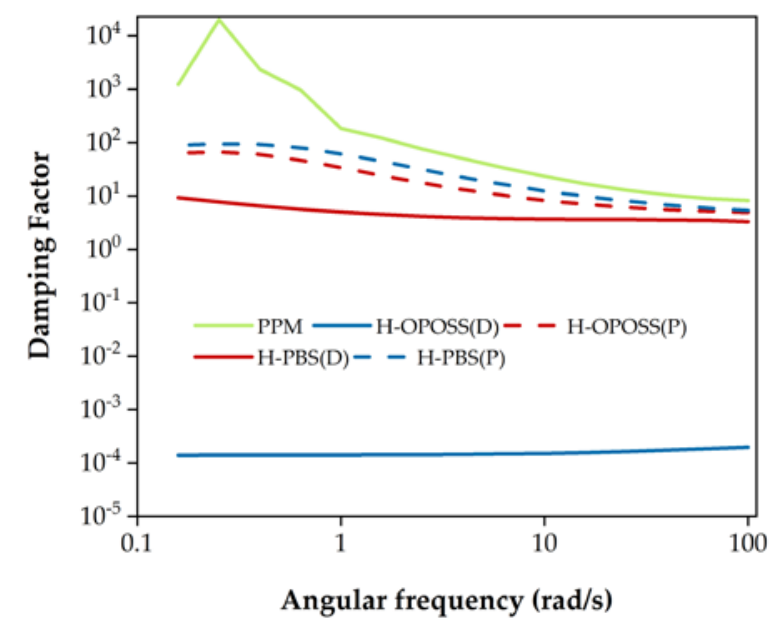

Figure 4. Damping factor of PPM without (green curve) and with additives (curves with other colors), as indicated in the figure.

Films with H-PBS(D) were prepared by hot pressing analogously to those described above with neat PPM. These materials were free of bubbles and formation of cracks was retarded (cracks emerged sometimes only after several hours or even after one whole day). In order to more efficiently prevent crack formation, a common plasticizer, benzyl butyl phthalate (BBP) was added to H-PBS(D), as the latter showed the highest viscosity (and still remained in a liquid-like condition). The addition of $17 \% \mathrm{~m} / \mathrm{m}$ BBP showed good results. It was not possible to measure viscosities of this system at $90{ }^{\circ} \mathrm{C}$ as in absence of BBP (see above) because the presence of BBP made the molten polymer too low viscous for the available equipment. Nonetheless it was possible to prepare films with this system by hot pressing at the same conditions as for the films above. These coatings with BBP were free of bubbles and cracks, also over periods at least for months. Figure 2 shows a coating two weeks after hot pressing. Accordingly, corrosion tests were performed with such films, designated as H-PBS(D)(BBP). The plasticizer significantly decreased the glass transition temperature of the PPM from 65 to $35^{\circ} \mathrm{C}$.

\subsection{Corrosion Tests}

The electrochemical characterization of coatings of H-PBS(D)(BBP) on silane treated AA2024 was performed by cyclic polarization scans in order to obtain information on the resistance of the coatings to pit attacks. Also, potentiostatic analyses were performed for the observation of the resistance of the coatings over time. For this purpose, a fixed potential was applied on the cell for $28 \mathrm{~h}$. The open circuit potential curves $(\mathrm{OCP})$ showed that at equilibrium conditions, the potential curves of the coated samples fit the values of the OCP curve of a blank AA2024 substrate (in the range of -0.6 and $-0.8 \mathrm{~V}$ related to SCE, in line with the literature [29]). The potential windows applied on the coated surfaces ranged from a -0.5 to $2.5 \mathrm{~V}$ (vs. SCE), and the corresponding current density was in the order of nanoampères, which confirms the barrier effect of the coating (Figure 5). This implies good anti-corrosion properties of the coating as the coating remained intact and the surface appeared uniform and compact, even when high potentials are reached. This fact is important as the efficiency of the coating relies on the separation of the metal surface from the corrosive environment. Thus, a physical barrier between metal surface and corrosive medium is established with the PPM-based coating. 


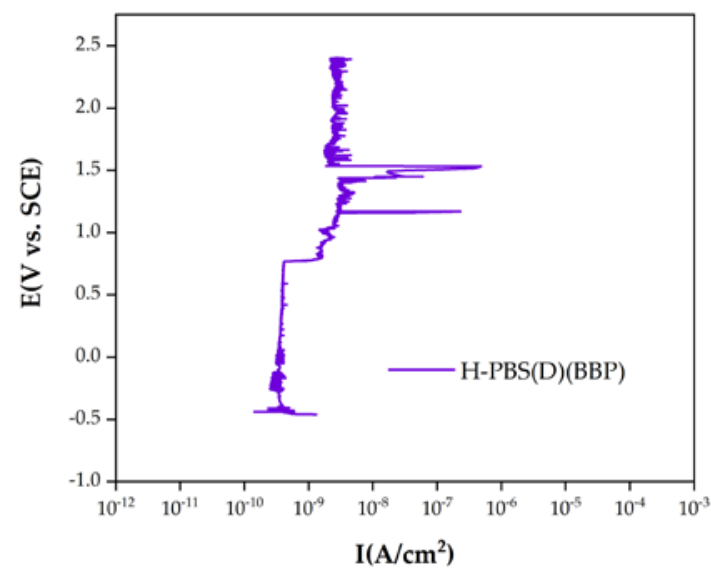

(a)

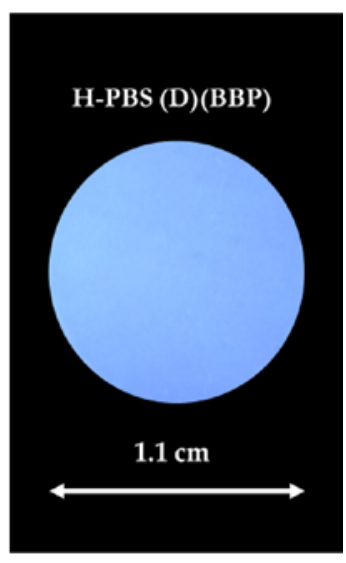

(b)

Figure 5. (a) Cyclic polarization curves of H-PBS(D)(BBP) coatings exposed to $0.6 \mathrm{~mol} \cdot \mathrm{L}^{-1}$ aqueous $\mathrm{NaCl}$ solution. (b) Surface of a coating exposed to the corrosion medium (picture taken at irradiation of light with wavelength of $365 \mathrm{~nm}$, causing the characteristic blue fluorescence of PPM).

Testing the stability of anti-corrosion coatings over extended periods is important in order to evaluate the suitability for future applications. Therefore potentiostatic analysis was performed on coated surfaces, applying a constant potential at which the coating was stable according to the previous anodic polarization measurement, namely at about $-0.3 \mathrm{~V}$ (cf. Figure 6). The results of this analysis shown in Figure 6 clearly demonstrate the resistance of the coating towards corrosion initiation over periods of at least $28 \mathrm{~h}$. This stability is in the high-end of coatings investigated with this method. Remarkably, a very sharp peak occurred at ca. $5.5 \mathrm{~h}$ (Figure 6), after which the initial current intensity was re-established again. This shows that a metastable pit stopped rapidly (after a few minutes), thus demonstrating the ability of the H-PBS(D)(BBP) to self-regenerate under pit attacks, i.e., to stop the beginning of corrosion. Notably, the coatings looked still homogeneous after the potentiostatic experiments, in particular no evidence of propagating corrosion was visible.

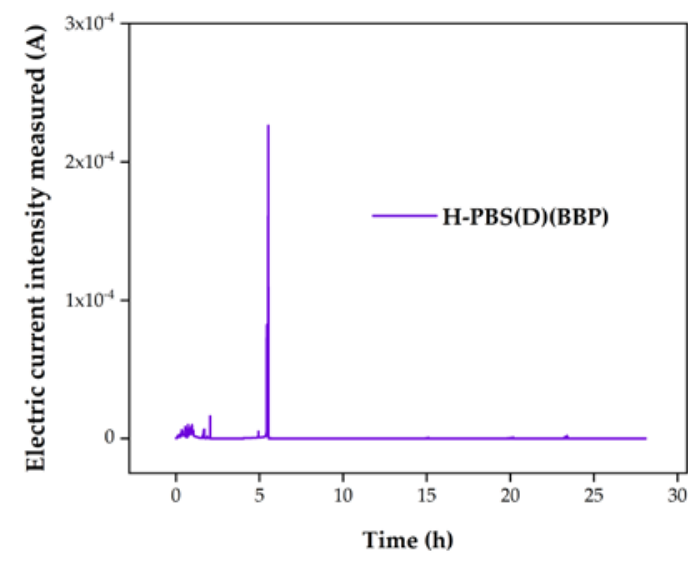

(a)

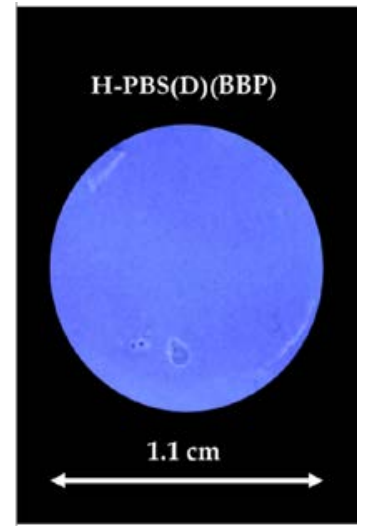

(b)

Figure 6. (a) Potentiostatic scan, at a constant potential of about $-0.3 \mathrm{~V}$, of H-PBS(D)(BBP)-coated substrates exposed to $0.6 \mathrm{~mol} \cdot \mathrm{L}^{-1} \mathrm{NaCl}$ solution. (b) State of the H-PBS(D)(BBP) surface after the scan: Surface of a coating exposed to the corrosion medium for $28 \mathrm{~h}$ (picture taken at irradiation of light with wavelength of $365 \mathrm{~nm}$, causing the characteristic blue fluorescence of PPM).

Corrosion inhibition may also be fostered by the pronounced hydrophobic nature of the coatings. The advancing contact angle of water on H-PBS(D)(BBP) layers amounted to $105^{\circ}$ and the receding 
contact angle to $98^{\circ}$, i.e., the contact angle hysteresis is remarkably low $\left(7^{\circ}\right)$. Notably the contact angle hysteresis was even lower than that on AA2024 surfaces which amounted to $14^{\circ}$, according to the advancing and receding contact angles of $55^{\circ}$ and $41^{\circ}$; respectively. The aluminum surfaces were not fully hydrophilic due to inevitable adsorption of atmospheric contaminants which proceeds within minutes [30,31]. As the contact angle hysteresis is an indication of surface homogeneity, it appears that the coated surfaces are even more homogeneous than the aluminum surface itself.

The electrochemical results of measured current densities, although limited to anodic polarization potentiodynamic analyses, allow to highlight a favorable protective behavior to corrosion by the PPM coating if compared to other organic coatings analyzed using the same electrochemical technique $[32,33]$.

\section{Discussion}

Basically, poly(phenylene methylene) (PPM) is an attractive polymer for studies in the area of coatings since it is thermally stable, hydrophobic and fluorescent; the latter facilitates optical detection of inhomogeneities and cracks by observation under UV light. Moreover it can readily be prepared in large quantities (100 $\mathrm{g}$ and more) also on the laboratory scale, in a straightforward process. In spite of this, coatings of PPM have hitherto not been investigated, because the isolation of sufficiently pure PPM of considerable molar mass $\left(10,000 \mathrm{~g} \cdot \mathrm{mol}^{-1}\right)$ was reported only recently. Therefore, the primary goal of this study was to explore the feasibility of PPM for formation of coatings, on the example of a metal substrate which is commonly used for corrosion tests (AA2024).

Several problems were identified in the preparation of coatings with PPM: Formation of bubbles, formation of cracks and detachment of PPM from the substrate. The latter was assumed to originate in the incompatibility of the highly apolar PPM and the highly polar surface of the AA2024 substrate. Already freshly polished AA2024 surfaces contain hydroxyl groups (evident from the symmetric and asymmetric $\mathrm{Al}-\mathrm{OH}$ bending vibrations of boehmite detected in infrared spectra) [34]. Surface hydroxyl groups are commonly converted with trialkoxysilanes $[23,24], \mathrm{RSi}\left(\mathrm{OR}^{\prime}\right)_{3}$, in presence of some water which can also stem from ambient humidity. The trialkoxysilanes can react both with surface hydroxyl groups and water, thus forming a thin and highly crosslinked surface-bound polysiloxane layer under release of the alcohol HOR'. The organic group $\mathrm{R}$ is still present in that layer and should be compatible with the coating. Here $\mathrm{R}$ is a benzyl group which corresponds closely to the structure of the constitutional repeat units of PPM. Indeed, the respective in-situ-formed polysiloxane layers on AA2024 improved qualitatively adhesion of PPM to the substrate in an extent that detachment of PPM was not observed anymore.

The extent of bubble formation depended on the preparation method of the coating and the presence of additives. Hot pressing of PPM powders led to less bubbles in the coating than deposition of dissolved PPM followed by solvent evaporation. Gratifyingly, powder processing is also a more straightforward method than processing from solution because the dissolution step is not necessary in powder processing. In addition, powder processing is faster because in particular the evaporation of solvent in the solution process proceeds slowly.

Eventually, bubbles could be suppressed efficiently by addition of $1 \% \mathrm{~m} / \mathrm{m}$ of PBS, an isolated polybenzylsiloxane, in coatings prepared by hot pressing, which was associated with a high increase in viscosity caused by the PBS. Accordingly, the interaction between the polymer chains is strong, thus apparently overcoming the energy required for bubble formation. PBS also retarded crack formation. This might be related to the stress accumulated to physical aging [35] during the cooling process after hot pressing. During ageing the free volume is reduced which is associated with a macroscopic volume contraction. This process can lead to widening of existing microcracks. It is common that such processes proceed within hours or days. Notably, fluorescence of PPM was preserved in presence of PBS, i.e., the PBS did not act as fluorescence quencher. In order to prevent cracks in a sustainable way, a plasticizer had to be added (BBP). In general, plasticizers enhance the elasticity of the system, and hence the system can adapt better to deformations which occur e.g., 
upon cooling the PPM-based coatings prepared by hot pressing. Viscosity is crucial to prevent bubbles, which can also lead to crack formation. In addition, crack formation can be caused by other factors such as internal stress inside the polymeric matrix. The latter can arise as a consequence of external stress occurring during film preparation (i.e., hot press) and the subsequent cooling process. A high elastic modulus and correspondingly a lower glass transition temperature $\left(T_{\mathrm{g}}\right)$ are helpful to attenuate those internal forces providing more mechanical stability to the coating. Thus, a compromise between the elastic modulus and the viscous modulus seems to be helpful in preventing bubbles and to mitigate internal force in the material.

Remarkably, electrochemical characterization revealed pronounced anti-corrosion properties of those PPM-based coatings, exhibiting a passive-like behavior in a wide potential window and a very low current density. This effect might be supported by the hydrophobic nature of the coating (advancing contact angle of water $105^{\circ}$ ), which may make the penetration of water to the metal substrate more difficult. Moreover, self-healing of the coating to pit attacks was found. Obviously, neat PPM is brittle below its glass transition temperature (about $65^{\circ} \mathrm{C}$ ). The stronger thermal shrinkage compared to the aluminum leads to tensile stresses, which cannot be taken by the PPM, due to missing mobility of the polymer chains. Since the plasticizer decreases the glass transition temperature, the chains regain their mobility and may thus close deficient locations, e.g., under the action of a pit attack.

With regard to future work, it could be explored if substituted PPMs show less of a tendency for crack formation, thus avoiding or at least reducing the need of additives for the generation of crack-free coatings. For instance, the synthesis of several methyl-substituted PPMs has already been reported $[4,21]$ but they have not been investigated yet for coatings. Further, fluorinated PPMs might attract attention since in general they are more hydrophobic than their hydrogenated analogues. With a view to the alternatives addressed above, the presented study on PPM itself can be regarded as an initiation of more investigations on the class of poly(phenylene methylene)s in the area of coatings.

Author Contributions: M.F.D. and A.B. performed the synthesis of the materials and the manufacture of the coatings under supervision of W.C. and M.N.; T.B.S. designed and contributed to the rheologic measurements which were performed together with M.F.D.; the corrosion tests which were performed by M.F.D. under supervision of S.P.M.T. and M.A.O. regarding the macromolecular features of the materials, and the manuscript was written by W.C. and M.F.D.

Funding: This research was funded by the Swiss National Science Foundation (No. 200021_159719/1).

Acknowledgments: We thank Elena Tervoort for taking SEM images.

Conflicts of Interest: The authors declare no conflict of interest.

\section{References}

1. Blilncow, P.J.; Pritchard, G. Determination of the structure of polyethylarylmethylenes by ${ }^{13} \mathrm{C}$ nuclear magnetic resonance spectroscopy. Polymer 1987, 28, 1824-1828. [CrossRef]

2. Ul Hasasn, M.; Tsonis, C.P. Structural characterization of polybenzyls by high field ${ }^{13} \mathrm{C}-\mathrm{NMR}$ spectroscopy. J. Polym. Sci. A Polym. Chem. 1984, 22, 1349-1355. [CrossRef]

3. Has, H.C.; Livingston, D.I.; Saunders, M. Polybenzyl type polymers. J. Polym. Sci. A Gen. Pap. 1955, 15, 503-514. [CrossRef]

4. Braendle, A.; Perevedentsev, A.; Cheetham, N.J.; Stavrinou, P.N.; Schachner, J.A.; Möosch-Zanetti, N.C.; Niederberger, M.; Caseri, W.R. Homoconjugation in poly(phenylene methylene)s: A case study of non- $\pi$-conjugated polymers with unexpected fluorescent properties. J. Polym. Sci. B Polym. Phys. 2017, 55, 707-720. [CrossRef]

5. Muller, P. Glossary of terms used in physical organic chemistry (IUPAC Recommendations 1994). Pure Appl. Chem. 1994, 306, 1077-1184. [CrossRef]

6. Ferguson, L.N.; Nnadi, J.C. Electronic interactions between nonconjugated groups. J. Chem. Educ. 1965, 42, 529-535. [CrossRef] 
7. Baumberger, T.R.; Woolsey, N.F. Metal arene complexation of polybenzyl: Preparation and methylation. J. Polym. Sci. A Polym. Chem. 1992, 30, 1717-1723. [CrossRef]

8. Dreyer, D.R.; Jarvis, K.A.; Ferreira, P.J.; Bielawski, C.W. Graphite oxide as a dehydrative polymerization catalyst: A one-step synthesis of carbon-reinforced poly(phenylene methylene) composites. Macromolecules 2011, 44, 7659-7667. [CrossRef]

9. Grassie, N.; Meldrum, I.G. Friedel-crafts polymers-IX Later stages of the copolymerization of p-di(chloromethyl)benzene with aromatic substances. Eur. Polym. J. 1971, 7, 1253-1273. [CrossRef]

10. Gunes, D.; Yagci, Y.; Bicak, N. Synthesis of soluble poly(p-phenylene methylene) from tribenzylborate by acid-catalyzed polymerization. Macromolecules 2010, 43, 7993-7997. [CrossRef]

11. Arata, K.; Fukui, A. Toyoshima High catalytic activity of calcined iron sulphates for the polycondensation of benzyl chloride. J. Chem. Soc. Chem. Commun. 1978, 121-122. [CrossRef]

12. Finocchiaro, P. Frazionamento di polimeri benzilici ottenuti a bassa temperature. Boll. Sci. Fac. Chim. Ind. Bologna 1968, 26, 255.

13. Hino, M.; Arata, K. Iron oxide as an effective catalyst for the polycondensation of benzyl chloride, the formation of para-substituted polybenzyl. Chem. Lett. 1979, 8, 1141-1144. [CrossRef]

14. Grassie, N.; Meldrum, I.G. Friedel-crafts polymers-2 Initial stages of the copolymerization of p-di(chloromethyl) benzene with benzene. Eur. Polym. J. 1969, 5, 195-209. [CrossRef]

15. Tsonis, C.P. Homogeneous catalytic polymerization of benzyl chloride leading to linear high molecular weight polymers: An elusive goal. J. Mol. Catal. 1990, 57, 313-323. [CrossRef]

16. Banks, R.E.; François, P.-Y.; Preston, P.N. Polymerization of benzyl alcohol in anhydrous hydrogen fluoride: An efficient synthesis of poly(phenylenemethylene). Polymer 1992, 33, 3974-3975. [CrossRef]

17. Klärner, C.; Greiner, A. Synthesis of polybenzyls by Suzuki Pd-catalyzed crosscoupling of boronic acids and benzyl bromides: Model reactions and polyreactions. Macromol. Rapid Commun. 1998, 19, 605-608. [CrossRef]

18. Som, A.; Ramakrishnan, S. Linear soluble polybenzyls. J. Polym. Sci. A Polym. Chem. 2003, 41, $2345-2353$. [CrossRef]

19. Saliba, P.A.; Mansus, A.A.; Santos, D.B.; Mansur, H.S. Fusion-bonded epoxy composite coatings on chemically functionalized API steel surfaces for potential deep-water petroleum exploration. Appl. Adhes. Sci. 2015, 3, 22. [CrossRef]

20. Choi, Y.K.; Hyeong, J.K.; Sung, R.K.; Young, M.C.; Dong, J.A. Enhanced Thermal Stability of Polyaniline with Polymerizable Dopants. Macromolecules 2017, 50, 3164-3170. [CrossRef]

21. Braendle, A.; Schwendimann, P.; Niederberger, M.; Caseri, W.R. Synthesis and fractionation of poly(phenylene methylene). J. Polym. Sci. A Polym. Chem. 2018, 56, 309-318. [CrossRef]

22. Twite, R.L.; Bioerwagen, G.P. Review of alternatives to chromate for corrosion protection of aluminum aerospace alloys. Prog. Org. Coat. 1998, 33, 91-100. [CrossRef]

23. Witucki, G.L. A Silane Primer: Chemistry and applications of alkoxy silanes. J. Coat. Technol. 1993, 65, 57-60.

24. Plueddemann, E.P. Chemistry of Silane Coupling Agents. In Silane Coupling Agents, 2nd ed.; Plueddemann, E.P., Ed.; Springer: Boston, MA, USA, 1991; pp. 31-54.

25. Goldschmidt, P.D.A.; Streitberger, D.H.-J. Basics of Coating Technology; Vincentz: Hannover, Germany, 2007.

26. Gilleo, K.B. Rheology and Surface Chemistry. In Coating Technology, Fundamentals, Testing and Processing Techniques; Tracton, A.A., Ed.; Taylor and Francis: Boca Raton, FL, USA, 2007; pp. 1-1-1-12.

27. Chan, C.-M.; Venkatraman, S. Coating Rheology. In Coating Technology, Fundamentals, Testing and Processing Techniques; Tracton, A.A., Ed.; Taylor and Francis: Boca Raton, FL, USA, 2007; pp. 2-1-2-14.

28. Kickelbick, G. Introduction to hybrid materials. In Hybrid Materials. Synthesis, Characterization, and Applications; Kickelbick, G., Ed.; WILEY-VCH Verlag GmbH \& Co. KGaA: Weinheim, Germany, 2007; pp. 1-46.

29. Trdan, U.; Grum, J. Evaluation of corrosion resistance of AA6082-T651 aluminium alloy after laser shockpeening by means of cyclic polarisation and ElS methods. Corros. Sci. 2012, 59, 324-333. [CrossRef]

30. Bain, C.D.; Troughton, E.B.; Tao, Y.-T.; Evall, J.; Whitesides, G.M.; Nuzzo, R.G. Formation of monolayer films by the spontaneous assembly of organic thiols from solution onto gold. J. Am. Chem. Soc. 1989, 111, 321-335. [CrossRef] 
31. Troughton, E.B.; Bain, C.D.; Whitesides, G.M.; Nuzzo, R.G.; Allara, D.L.; Porter, M.D. Monolayer films prepared by the spontaneous self-assembly of symmetrical and unsymmetrical dialkyl sulfides from solution onto gold substrates: Structure, properties, and reactivity of constituent functional groups. Langmuir 1988, 4, 365-385. [CrossRef]

32. Talo, A.; Passiniemi, P.; Forsén, O.; Yläsaari, S. Polyaniline/Epoxy Coatings with Good Anti-Corrosion Properties. Synth. Met. 1997, 85, 1333-1334. [CrossRef]

33. Rodošek, M.; Rauter, A.; Perše, L.S.; Kek, D.M.; Vuk, A.S. Vibrational and corrosion properties of poly(dimethylsiloxane)-based protective coatings for AA 2024 modified with nanosized polyhedral oligomeric silsesquioxane. Corros. Sci. 2014, 85, 193-203. [CrossRef]

34. Kreta, A.; Rodošek, M.; Perše, L.S.; Orel, B.; Gaberšček, M.; Vuk, A.Š. In situ electrochemical AFM, ex situ IR reflection-absorption and confocal Raman studies of corrosion processes of AA 2024-T3. Corros. Sci. 2016, 104, 290-309. [CrossRef]

35. Jin, K.; Li, L.; Torkelson, J.M. Bulk physical aging behavior of cross-linked polystyrene compared to its linear precursor: Effects of cross-linking and aging temperature. Polymer 2017, 115, 197-203. [CrossRef]

(C) 2018 by the authors. Licensee MDPI, Basel, Switzerland. This article is an open access article distributed under the terms and conditions of the Creative Commons Attribution (CC BY) license (http:/ / creativecommons.org/licenses/by/4.0/). 